ORIGINAL ARTICLE

\title{
Timing of routine immunisations and subsequent hay fever risk
}

\author{
S A Bremner, I M Carey, S DeWilde, N Richards, W C Maier, S R Hilton, D P Strachan, D G Cook
}

Arch Dis Child 2005;90:567-573. doi: 10.1136/adc.2004.051714

See end of article for authors' affiliations

Correspondence to: Prof. D G Cook, Professor of Epidemiology, Department of Community Health Sciences, St

George's Hospital Medical School, Cranmer Terrace, London SW17 ORE, UK; d.cook@sghms.ac.uk

Accepted 6 July 2004
Background: Suggestions that immunisation influences allergic disease risk, either positively (pertussis) or negatively (BCG) are of concern for vaccination policy.

Aims: To determine whether DTP, MMR, and BCG vaccination in infancy influenced hay fever risk.

Methods: Case-control study of 7098 hay fever cases and controls, within two primary care databases. One control per case was matched for practice, age, and sex. Odds ratios (OR) were derived using conditional logistic regression.

Results: Compared to those completing in month 5 (base group) (39.3\%), DTP unvaccinated children (4.3\%) had a similar risk of hay fever (OR $=0.94,95 \% \mathrm{Cl} 0.73$ to 1.23). However, those completing after 12 months (4.2\%) had a reduced risk $(O R=0.60,95 \% \mathrm{Cl} 0.45$ to 0.76$)$ compared to the base group. Compared to those vaccinated in month 14 (base group) (29.5\%), MMR unvaccinated children (2.3\%) had an OR of 0.79 (95\% $\mathrm{Cl} 0.58$ to 1.08). Completion of MMR after two years was associated with reduced hay fever risk $(O R=0.62,95 \% \mathrm{Cl} 0.48$ to 0.80$)$ compared to the base group. The effects of late immunisation with DTP and MMR were independent. Those vaccinated with BCG by age $2(2.4 \%)$ had an odds ratio of 1.28 (95\% Cl 0.96 to 1.70). Adjustment for consulting behaviour, social factors, or sibship size did not alter these associations.

Conclusions: Immunisation against DTP or MMR does not increase the risk of hay fever. The lower confidence limit for BCG vaccination contradicts the hypothesised protective effect. The reduced risk of hay fever among children immunised late may be explained by a third factor causing both postponement and reduced risk such as intercurrent febrile illnesses.
A rise in the prevalence of atopic diseases such as allergic rhinitis during recent decades has been widely reported from developed countries. ${ }^{1-3}$ One postulated explanation is the reduction in childhood infections due to successful vaccination programmes. Without the challenge of potentially serious infection to trigger a Thl immune response, a Th2 response could predominate in the developing immune system, predisposing to atopy and allergic diseases. The literature on infections and atopic disease is equivocal. ${ }^{4-7}$ However, vaccines themselves may influence the infantile immune system by shifting expression in favour of either Thl or Th2 cytokine profiles. ${ }^{89}$ The relevant epidemiological evidence is inconsistent. ${ }^{9}$ Although one recent review concluded that the evidence did not support vaccines causing allergic diseases, it focussed primarily on studies where the outcome was asthma, a disease which has both atopic and non-atopic components, particularly in early infancy. ${ }^{10}$ Studies focusing on allergic rhinitis provide a more specific test of the hypothesis.

DTP vaccination (against diphtheria, tetanus, and pertussis) has been considered as a possible inducer of immunoglobulin E production through the whole cell pertussis component. While positive associations have been reported with atopic disease, ${ }^{11}$ allergy related respiratory symptoms, ${ }^{12}$ and allergic illnesses, ${ }^{13}$ other studies have found no association between DTP vaccination and allergic diseases, ${ }^{14}$ hay fever, ${ }^{15}$ or atopy assessed by skin prick tests. ${ }^{16}{ }^{17}$

The triple vaccine against mumps, measles, and rubella (MMR) has also been hypothesised as a risk factor for atopy, mainly due to the measles component, but again the evidence is inconsistent. Wild measles infection was associated with a lower prevalence of allergic sensitisation in a study set in Guinea Bissau, ${ }^{18}$ while a Finnish study found that children infected with wild measles were at an increased risk of later allergic rhinitis. ${ }^{19}$ Not receiving MMR vaccination was protective of atopy in a study set in Steiner schools in Sweden. ${ }^{20}$ The Guinean and Swedish studies have been criticised for uncontrolled confounding. However, a recent study of vaccinations and early onset hay fever using the West Midlands section of the General Practice Research Database showed that, after controlling for consultation behaviour, the apparent protective effect against hay fever of vaccination with MMR mostly disappeared..$^{14}$ A study set in five German cities found a negative dose-response relation between number of vaccine doses (MMR and DTP among others) and allergic sensitisation. ${ }^{21}$ Finally, in one national British birth cohort, an inverse association between hay fever and measles immunisation and/or infection was apparently confined to those with many older siblings. ${ }^{22}$

Being a live mycobacterial vaccine, BCG (bacillus CalmetteGuérin) activates a strong Thl response. Following the observation that tuberculin reactivity was strongly inversely related to IgE levels ${ }^{23}$ it has been suggested that this may protect against a later atopic response, and BCG has been associated with lower risk of subsequent atopy in some studies, ${ }^{2425}$ but not in others. ${ }^{26-30}$

To clarify the epidemiological evidence, we conducted nested case-control studies in two large UK databases of primary care consultations (the General Practice Research Database (GPRD) and the Doctors' Independent Network (DIN)) to test the hypotheses that: (1) immunisation with DTP in the first year of life increases subsequent hay fever risk; (2) receipt of MMR vaccine in the first two years of life increases subsequent hay fever risk; and (3) BCG vaccination before age 2 years protects against subsequent hay fever. We paid particular attention to the timing of each vaccination in order to identify any critical period for long term immunological programming. 


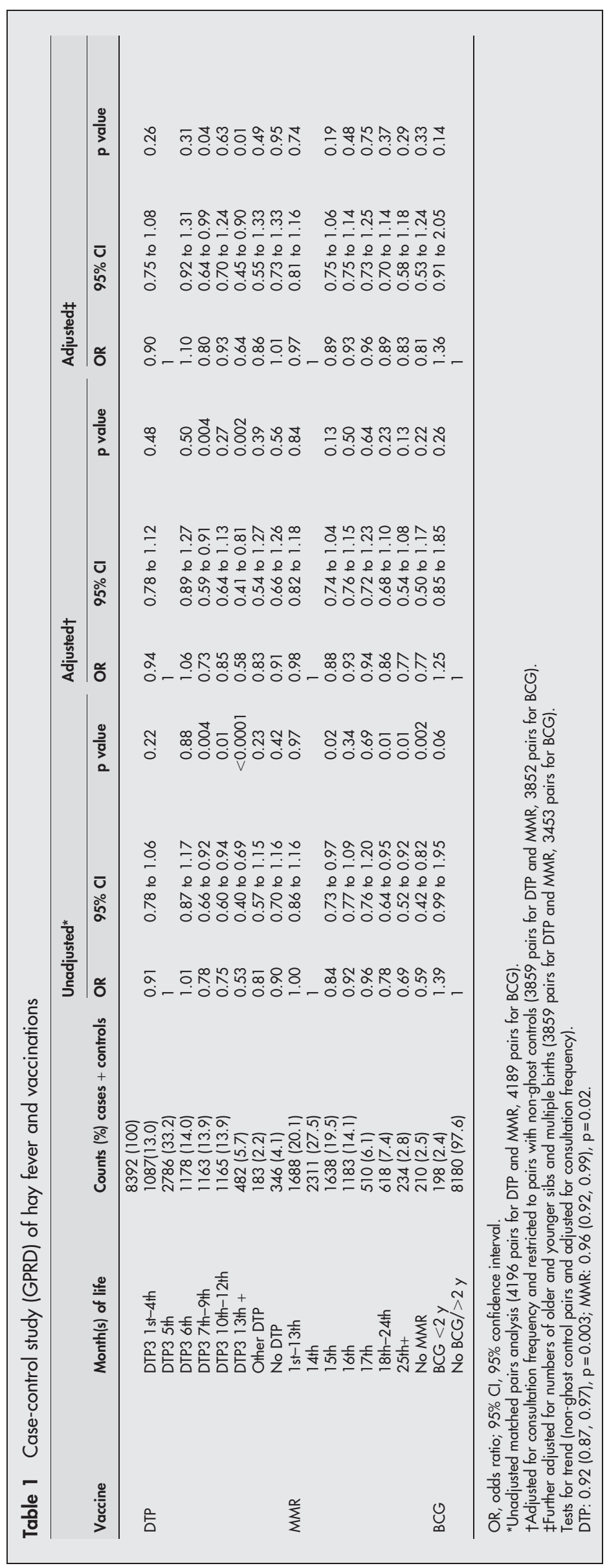

\section{METHODS}

A detailed description of our methodology has been published previously. ${ }^{31}$ In summary, we conducted nested case-control studies within a GPRD cohort of 76310 children born between 1989 and 1993 from 464 general practices, and within a DIN cohort of 40183 children born between 1989 and 1997 from 141 general practices. All children were registered with a contributing practice within three months of birth and had at least five years of continuous follow up with that practice. Practices were located primarily in England and Wales but Scotland and Northern Ireland were also represented.

We aimed to be consistent across the two databases in our case definition of hay fever and selection of relevant drugs, despite the various coding systems used. Only codes synonymous with "allergic rhinitis" and with seasonal variation in recording were permitted. To assess caseness, sequential diagnoses of hay fever and/or specific medications were sought. Subjects who only ever had a hay fever diagnosis before age 2 , or non-specific allergic rhinitis diagnoses which did not exhibit a seasonal pattern were excluded from being cases or controls. All cases were required to have at least one diagnosis of hay fever. For each case of hay fever we selected one control child, matched for general practice, sex, and birth month who had continuous follow up until at least the date of diagnosis of the index case. ${ }^{31}$ Cases and controls were thus followed up for similar lengths of time: 7.42 and 7.43 years respectively in GPRD and 8.22 and 8.24 years in DIN.

Records of cases and controls from GPRD (4196 pairs) and DIN (2902 pairs) were searched for the first occurrence of 3rd DTP immunisation (DTP3 with or without Haemophilus influenzae type b and/or poliomyelitis) or if absent, any other entries for DTP or pertussis. We chose 3rd DTP for the main exposure as some general practices did not routinely record 1 st and 2nd doses. We also identified first entries for MMR immunisation.

The sensitivity of any relation between hay fever and DTP, MMR, and BCG was assessed by controlling for consultation frequency in the first five years of life, determined by categorising the number of consultations during that time, excluding days on which vaccinations were administered.

In the UK during the 1990s, DTP (whole cell pertussis) vaccine was normally administered adsorbed onto a mineral compound. It was routinely given as three separate injections at 8 , 12 , and 16 weeks. ${ }^{32}$ It is usually administered at the same time as an oral live poliomyelitis vaccine and can also be given in combination with Haemophilus influenzae type B vaccine (HiB). On its introduction, MMR-II was recommended in year 2 and subsequently in months 12-15. BCG vaccination is not routine during infancy, but is generally reserved for neonates at high risk of tuberculosis. At the time of our study, the Copenhagen substrain 1077 of BCG, given intradermally in a dose of $0.05 \mathrm{ml}$ (to infants under 3 months of age) or $0.1 \mathrm{ml}$ was recommended.

For the principal analyses, timing of DTP3 was categorised as 1st-4th month, 5th, 6th, 7th-9th, 


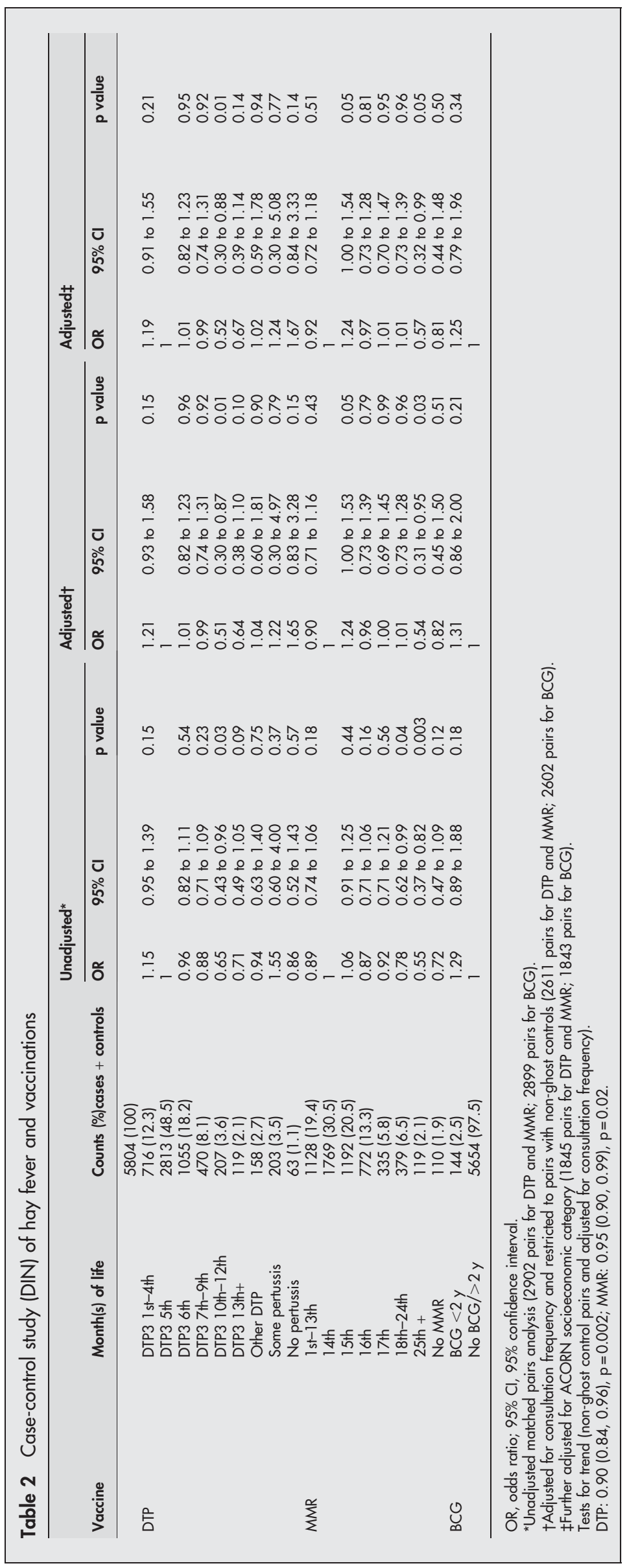

10th-12th, 13th or later, DTP other than 3rd, pertussis (single vaccination) (DIN only), and unvaccinated against pertussis. MMR was categorised into the time bands: 1st-13th month, 14th, 15th, 16th, 17th, 18th-24th, and 25th month or later. Odds ratios are reported with those vaccinated "on time" (modal group) forming the reference group (month 5 for DTP3 and month 14 for MMR).

Finally, records of BCG vaccination were sought in birth cohort members during their first two years of follow up. Our main exposure period of interest was BCG by the age of 2 years. Those vaccinated with BCG after the age of 2 were classed as unvaccinated for the purpose of this analysis. Case-control pairs where either member had a record of tuberculosis at any age were excluded.

\section{Statistical analysis}

Our analyses are based on conditional multiple logistic regression models, with the matched pairs forming separate strata, using proc phreg in SAS (version 8.1 for SunOS). ${ }^{33}$

Within each database we adjusted for consultation frequency and examined the effect of excluding potential "ghost" controls. These were children who had no record of a general practitioner consultation in the period six months before the case diagnosis date or at any time after. The true disease and exposure status of such controls would be unknown but we might expect some of them to be misclassified cases. We also investigated the possible confounding influence of socioeconomic circumstances measured at the small area level by ACORN classification in $\mathrm{DIN}^{34}$ and sibship structure based on the family identifier in GPRD.

The odds ratio estimates from the two casecontrol studies excluding potential "ghost" controls and adjusted for consultation frequency were pooled using a fixed effects model by way of the meta command in Stata (version 5.0 for Windows). ${ }^{35}{ }^{36}$ In pooled, fixed effects models, we explored whether the effects of DTP/ MMR were independent of each other and whether there was any evidence of a trend across categories of timing of vaccination.

\section{RESULTS}

DTP

In GPRD, $93.7 \%$ of cases plus controls were fully vaccinated with DTP, while only $4.1 \%$ were entirely unvaccinated (table 1). In DIN, $92.8 \%$ were fully DTP vaccinated and only 1.1\% were entirely unvaccinated, with $3.5 \%$ receiving pertussis only (table 2 ).

In both databases the DTP unvaccinated (also pertussis unvaccinated in DIN) group were at very similar risk of subsequent hay fever to those vaccinated "on time" with unadjusted odds ratios of 0.90 in GPRD (table 1) and 0.86 in DIN (table 2). Children identified as having incomplete or non-specific DTP records were small in number and there was no evidence of an excess or a deficit risk in this group. However, later/delayed vaccination with DTP3 was associated with decreased risk of hay fever, 
and the trend was significant in both databases. In both databases adjusting for consultation frequency and restricting to non-ghost controls had little effect on the odds ratio estimated, save for the "no pertussis" group in DIN. In GPRD, the number of older sibs was inversely related to hay fever risk such that for each additional older sib, the odds of hay fever reduced by $17.3 \%$ (12.9-21.4\%). Further adjustment for sibship structure was possible for $90 \%$ of case-control pairs in GPRD, but had little impact on the odds ratios (table 1). In DIN, the ACORN index was not significantly associated with hay fever risk: the odds ratio of hay fever was 0.99 (0.801.22 ) in the thriving ACORN group compared to the striving group. Not surprisingly, further adjustment for the ACORN socioeconomic index had little impact (table 2). The similarity of the trends in the two databases with late DTP3 vaccination is apparent in fig 1 .

\section{MMR}

In GPRD, $97.5 \%$ of cases plus controls were vaccinated with MMR, with $87.3 \%$ being vaccinated by 18 months, a further $7.4 \%$ by age 2 years, and $2.8 \%$ after age $2 ; 2.5 \%$ had no record of vaccination (table 1 ). In DIN, $98.1 \%$ of cases plus controls received MMR; $89.5 \%$ by 18 months, a further $6.5 \%$ by age 2 years, and $1.9 \%$ after age $2 ; 1.9 \%$ had no record of vaccination (table 2 ).

For MMR, the unvaccinated group were at lower risk of hay fever than those vaccinated on time: odds ratios of 0.59 in GPRD (table 1) and 0.72 in DIN (table 2). Again there was a decline in risk associated with later age of vaccination which was significant in both databases and the odds ratios for the unvaccinated were similar to those for those vaccinated with MMR after 18 months. Adjustment for consultation frequency and restricting to non-ghost controls had no major impact, but the odds ratio for the unvaccinated group in GPRD was less extreme after these adjustments $(\mathrm{OR}=0.77)$. Further adjustment for sibship structure (table 1) and ACORN socioeconomic score (table 2) made no impact on the odds ratios. The similarity of the results for the two databases is shown in fig 2.

Because the results are so similar for both GPRD and DIN, further analyses to assess the independence of the trends with delayed vaccination for the two triple vaccines were based on pooling estimates from the two databases (table 3). It is apparent that the trends are largely independent with the inverse association between hay fever and postponement being significant for both DTP $(p=0.001)$ and MMR $(\mathrm{p}=0.04)$.

\section{BCG}

The percentage of cases and controls vaccinated with BCG was almost identical in each database: $2.4 \%$ of GPRD and $2.5 \%$ of DIN had a record of vaccination by age $2,83 \%$ of these within the first three months of life (tables 1 and 2). A further $0.2 \%$ in each database were vaccinated by age 5 years.

In both databases immunisation with BCG by age 2 was associated with an increased risk of hay fever. The pooled estimate was 1.34 (95\% CI 1.05 to $1.73 ; \mathrm{p}=0.02)$ which reduced to 1.28 (95\% CI 0.96 to $1.73 ; \mathrm{p}=0.09$ ) after adjusting for consultation frequency and restriction to non-ghost controls.

\section{DISCUSSION}

In our large scale investigation we have found that infants immunised against DTP or MMR in accordance with vaccination schedules are at no greater or lesser risk of hay fever than those who do not receive these immunisations. This is reassuring in relation to the impact of routine vaccinations on atopic sensitisation, and concurs with the results of an international comparison of the prevalence of allergic diseases and vaccine coverage. ${ }^{37}$

The finding of a protective effect of late immunisation was unexpected, but was consistent in the two databases; a powerful indication that the findings are not artefacts of a specific method of recording. Moreover the odds of hay fever fall steadily with increasing lateness of vaccination for both DTP and MMR (figs 1 and 2) and the tests for trend are significant. The associations are therefore likely to be real, although not necessarily causal. We consider potential explanations under three headings: artefact, family factors, and childhood factors.

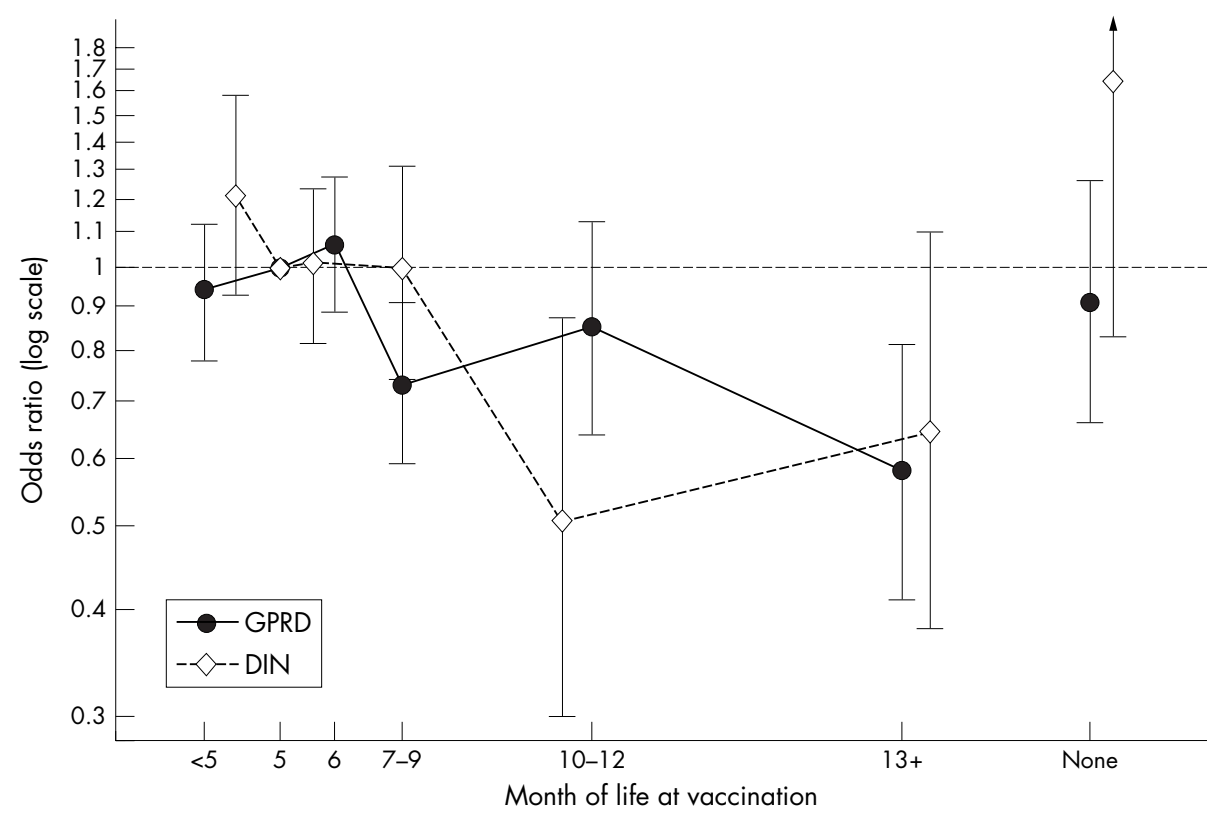

Figure 1 Odds of hay fever by age (in months) at 3rd DTP. Estimated odds ratios with $95 \% \mathrm{Cl}$. Arrow represents upper confidence limit off scale. Vaccination in month 5 is base group. None represents unvaccinated. 


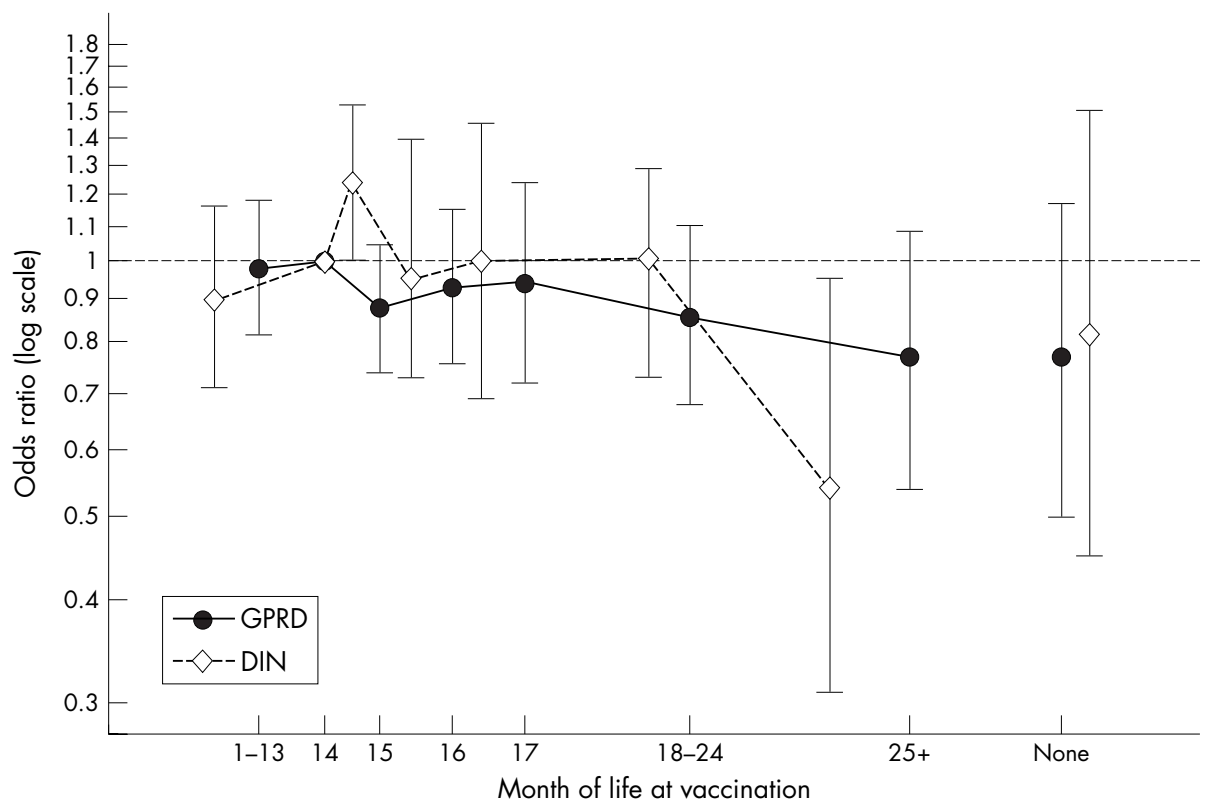

Figure 2 Odds of hay fever by age (in months) at MMR. Estimated odds ratios with $95 \% \mathrm{Cl}$. Vaccination in month 14 is base group. None represents unvaccinated.

\section{Artefact}

This is by far the largest and most comprehensive study to date of the association between immunisation history and subsequent allergic disease. Although delayed vaccinations were uncommon, the size of our study detects trends with high levels of statistical significance.

Restriction of potential "ghost" controls had no important impact on our results.

It is theoretically possible that families who consult their GP less often would be less likely to attend for routine vaccination and less likely to present their child with hay fever symptoms. However, adjustment for consultation frequency made little difference and there was no reduction in hay fever risk among DTP unvaccinated children, which makes this explanation unlikely.

\section{Family factors}

In GPRD, the numbers of older and younger sibs were available for $90 \%$ of case-control pairs. As consistently found elsewhere, children from larger families were less likely to complete vaccination on time, and having more older or younger sibs was strongly protective of hay fever. ${ }^{38}$ However, adjusting for sibship structure had no effect on the relation between hay fever and timing of vaccination (table 1).

For both triple vaccines in DIN, we found a lower percentage immunised on time in the more deprived ACORN groups and a greater percentage not immunised or immunised late. However, in regression models, ACORN was found not to confound the relation between exposure timing and later hay fever (table 2).

\section{Child factors}

Allergic children may differ from other children in several ways. Their more frequent contacts with their GP may mean they are also more likely to complete vaccinations. However, while consultation frequency was strongly positively related to the risk of hay fever, adjusting for it did not alter the odds ratios for hay fever by age at immunisation.

Table 3 Pooled (fixed effects) results for GPRD and DIN

\begin{tabular}{|c|c|c|c|c|c|c|c|}
\hline \multirow[b]{2}{*}{ Vaccine } & \multirow[b]{2}{*}{ Month(s) of life } & \multicolumn{3}{|c|}{ DTP and MMR modelled independently } & \multicolumn{3}{|c|}{ DTP and MMR modelled jointly } \\
\hline & & OR & $95 \% \mathrm{Cl}$ & p value & $\overline{O R}$ & $95 \% \mathrm{Cl}$ & p value \\
\hline \multirow[t]{7}{*}{ Age at DTP3 } & $\begin{array}{l}\text { DTP3 1st-4th } \\
\text { DTP3 5th }\end{array}$ & $\begin{array}{l}1.02 \\
1\end{array}$ & 0.90 to 1.17 & 0.74 & $\begin{array}{l}1.02 \\
1\end{array}$ & 0.89 to 1.17 & 0.78 \\
\hline & DTP3 6th & 0.99 & 0.88 to 1.12 & 0.92 & 1.00 & 0.89 to 1.13 & 0.996 \\
\hline & DTP3 7th-9th & 0.80 & 0.69 to 0.94 & 0.005 & 0.83 & 0.71 to 0.97 & 0.02 \\
\hline & DTP3 10th-12th & 0.72 & 0.58 to 0.90 & 0.005 & 0.75 & 0.60 to 0.94 & 0.01 \\
\hline & DTP3 13th + & 0.60 & 0.45 to 0.76 & $<0.001$ & 0.66 & 0.50 to 0.86 & 0.002 \\
\hline & Other DTP & 0.88 & 0.65 to 1.20 & 0.41 & 0.94 & 0.68 to 1.28 & 0.69 \\
\hline & No pertussis & 0.94 & 0.73 to 1.23 & 0.65 & 0.99 & 0.76 to 1.28 & 0.92 \\
\hline \multirow[t]{7}{*}{ Age at 1 st $M M R$} & $\begin{array}{l}1 \mathrm{st}-13 \mathrm{th} \\
14 \text { th }\end{array}$ & $\begin{array}{l}0.94 \\
1\end{array}$ & 0.83 to 1.07 & 0.36 & $\begin{array}{l}0.92 \\
1\end{array}$ & 0.81 to 1.05 & 0.24 \\
\hline & 15 th & 0.96 & 0.85 to 1.08 & 0.48 & 0.97 & 0.86 to 1.10 & 0.62 \\
\hline & 16 th & 0.89 & 0.76 to 1.04 & 0.13 & 0.91 & 0.79 to 1.06 & 0.24 \\
\hline & 17th & 0.90 & 0.74 to 1.10 & 0.31 & 0.94 & 0.77 to 1.15 & 0.56 \\
\hline & 18th-24th & 0.80 & 0.67 to 0.95 & 0.01 & 0.85 & 0.71 to 1.01 & 0.07 \\
\hline & 25 th + & 0.62 & 0.48 to 0.80 & $<0.001$ & 0.67 & 0.52 to 0.90 & 0.007 \\
\hline & No MMR & 0.79 & 0.58 to 1.08 & 0.13 & 0.82 & 0.59 to 1.12 & 0.21 \\
\hline
\end{tabular}

All of the analyses are based on non-ghost control pairs only and are adjusted for consultation frequency.

OR, odds ratio; $95 \% \mathrm{Cl}$, 95\% confidence interval. 


\section{What is already known on this topic}

- Routine vaccinations in early life offer essential protection against potentially fatal diseases

- Evidence for associations between atopic disease and the pertussis component of DTP and the measles component of MMR is inconclusive

A more plausible explanation for postponing immunisation would be presentation with a fever at the time an immunisation was due. If this was as a result of infection with a virus or bacterium which protected against subsequent development of hay fever, then postponement would indirectly be associated with lower risk. We propose that this is the most plausible explanation for our observation, but it will require further exploration and independent confirmation as details of reasons for postponing vaccinations were not recorded routinely on either database. A simple analysis of mean infection rate on a month-by-month basis in years 1 and 2 did not show a graded relation with vaccination delay. This is the subject of ongoing research.

However, it would be premature to suggest changes to vaccination policy on the basis of our findings. MMR and DTP protect against potentially fatal diseases and recent media scares in the UK may have contributed to a reduction in immunisation uptake in recent years. ${ }^{39}$

Finally, our finding that the small group of infants vaccinated with BCG in early life were at a small increased risk of hay fever was contrary to expectations. Again, this result was not explained in any way by confounding due to ACORN category or sibship structure. While the small increased risk of hay fever associated with BCG is not important, compared to the protection offered against tuberculosis, our results, with narrow confidence intervals, effectively rule out the hypothesised protective effect of BCG on subsequent risk of allergic disease.

\section{Conclusions}

In summary, this study shows that infants vaccinated with DTP and MMR are at no greater or lesser risk of developing hay fever than unvaccinated children. This should reassure parents and clinicians and no opportunity should be missed to immunise. Postponement of routine immunisation in early life is associated with a reduction in hay fever risk. This is a novel and potentially important observation which deserves to be further investigated.

There is an increasing risk of a measles epidemic. ${ }^{40}$ Delaying vaccination would further increase the number of susceptible subjects at younger ages and almost certainly increase the number of children not completing their immunisations.

We believe that the most likely explanation is that infections associated with fever both lead to postponement of immunisation and protect against development of atopy. If this interpretation is correct, it implies that common and relatively mild intercurrent illnesses which may not be presented to the GP, rather than serious infections, may underlie the "hygiene hypothesis" for allergic disease. ${ }^{3841}$

\footnotetext{
Authors' affiliations

S A Bremner, I M Carey, S DeWilde, S R Hilton, D P Strachan, D G Cook, Department of Community Health Sciences, St George's Hospital Medical School, London, UK

N Richards, CompuFile Limited, Send, Surrey, UK

W C Maier, Worldwide Epidemiology Group, GlaxoSmithKline, Greenford, Middlesex, UK
}

\section{What this study adds}

- Children vaccinated with DTP or MMR are at no greater risk of developing hay fever than those unvaccinated

- Late vaccinees are at a reduced risk of developing hay fever. This is not explained by measured social or behavioural indicators

- BCG vaccinated children are at a slightly increased risk of developing hay fever. This is contrary to expectations

- These findings are consistent in two large, independent samples in the UK

Funding: Wellcome Trust grant 065177/Z/01/Z

Competing interests: Nicky Richards is a director of CompuFile Ltd which markets DIN data to pharmaceutical companies.

\section{REFERENCES}

1 Burney P. The changing prevalence of asthma? Thorax 2002;57:1136-9.

2 Hopkin JM. The rise of atopy and links to infection. Allergy 2002;57:5-9.

3 Upton MN, McConnachie A, McSharry C, et al. Intergenerational 20 year trends in the prevalence of asthma and hay fever in adults: the Midspan family study surveys of parents and offspring. BMJ 2000;321:88

4 Benn CS, Melbye M, Wohlfahrt J, et al. Cohort study of sibling effect, infectious diseases, and risk of atopic dermatitis during first 18 months of life. BMJ 2004;328:1223.

5 McKeever TM, Lewis SA, Smith C, et al. Early exposure to infections and antibiotics and the incidence of allergic disease: a birth cohort study with the West Midlands General Practice Research Database. J Allergy Clin Immunol 2002; 109:43-50.

6 Matricardi PM, Ronchetti R. Are infections protecting from atopy? Curr Opin Allergy Clin Immunol 2001;1:413-19.

7 Matricardi PM, Rosmini F, Panetta V, et al. Hay fever and asthma in relation to markers of infection in the United States. J Allergy Clin Immunol 2002;110:381-7.

8 Grüber C, Nilsson L, Biörkstén B. Do early childhood immunizations influence the development of atopy and do they cause allergic reactions? Pediatr Allergy Immunol 2001;12:296-311.

9 Pershagen G. Can immunization affect the development of allergy? Pediatr Allergy Immunol 2000; 1 1 (suppl 13):26-8.

10 Offit PA, Hackett CJ. Addressing parents' concerns: do vaccines cause allergic or autoimmune diseases? Pediatrics 2003;111:653-9.

11 Faroogi IS, Hopkin JM. Early childhood infection and atopic disorder. Thorax 1998;53:927-32.

12 Hurwitz EL, Morgenstern H. Effects of diphtheria-tetanus-pertussis or tetanus vaccination on allergies and allergy-related respiratory symptoms among children and adolescents in the United States. J Manipulative Physiol Ther 2000;23:81-90.

13 Kemp T, Pearce N, Fitzharris $P$, et al. Is infant immunization a risk factor for childhood asthma or allergy? Epidemiology 1997;8:678-80.

14 McKeever TM. A birth cohort study of the aetiology of allergic disease in children using the West Midlands General Practice Research Database. PhD thesis. University of Nottingham, 2002.

15 Wist $M$, Dold S, Reitmeir $P$, et al. Pertussis infection and allergic sensitization. Ann Allergy 1994;73:450-4.

16 Maitra A, Sherriff A, Griffiths $M$, et al. Pertussis vaccination in infancy and asthma or allergy in later childhood: birth cohort study. BMJ 2004;328:925-6.

17 Nilsson L, Kjellman NI, Björkstén B. Allergic disease at the age of 7 years after pertussis vaccination in infancy: results from the follow-up of a randomized controlled trial of 3 vaccines. Arch Pediatr Adolesc Med 2003;157:1 184-9

18 Shaheen SO, Aaby P, Hall AJ, et al. Measles and atopy in Guinea-Bissau. Lancet 1996;347:1792-6.

19 Paunio M, Heinonen OP, Virtanen M, et al. Measles history and atopic diseases: a population-based cross-sectional study. JAMA 2000;283:343-6.

20 Alm JS, Swartz J, Lilja G, et al. Atopy in children of families with an anthroposophic lifestyle. Lancet 1999;353:1485-8.

21 Grüber C, Illi S, Lau S, et al. Transient suppression of atopy in early childhood is associated with high vaccination coverage. Pediatrics 2003;111:E282-8.

22 Lewis SA, Britton JR. Measles infection, measles vaccination and the effect of birth order in the aetiology of hay fever. Clin Exp Allergy 1998:28:1493-500.

23 Shirakawa T, Enomoto T, Shimazu S, et al. The inverse association between tuberculin responses and atopic disorder. Science 1997;275:77-9.

24 Aaby $\mathrm{P}$, Shaheen $\mathrm{SO}$, Heyes $\mathrm{CB}$, et al. Early BCG vaccination and reduction in atopy in Guinea-Bissau. Clin Exp Allergy 2000;30:644-50. 
25 Grüber C, Meinlschmidt G, Bergmann R, et al. Is early BCG vaccination associated with less atopic disease? An epidemiological study in German preschool children with different ethnic backgrounds. Pediatr Allergy Immunol 2002;13:177-81.

26 Krause TG, Hviid A, Koch A, et al. BCG vaccination and risk of atopy. JAMA 2003;289:1012-15

27 Wong GW, Hui DS, Tam CM, et al. Asthma, atopy and tuberculin responses in Chinese schoolchildren in Hong Kong. Thorax 2001;56:770-3.

28 Alm JS, Lilja G, Pershagen G, et al. Early BCG vaccination and development of atopy. Lancet 1997;350:400-3.

29 Strannegård IL, Larsson LO, Wennergren G, et al. Prevalence of allergy in children in relation to prior BCG vaccination and infection with atypical mycobacteria. Allergy 1998;53:249-54.

30 Grüber C, Kulig M, Bergmann R, et al. Delayed hypersensitivity to tuberculin, total immunoglobulin $\mathrm{E}$, specific sensitization, and atopic manifestation in longitudinally followed early bacille Calmette-Guerin-vaccinated and nonvaccinated children. Pediatrics 2001;107:e36.

31 Bremner SA, Carey IM, DeWilde S, et al. Early-life exposure to antibacterials and the subsequent development of hayfever in childhood in the UK: casecontrol studies using the General Practice Research Database and the Doctors' Independent Network. Clin Exp Allergy 2003;33:1518-25.
32 Department of Health, Welsh Office, Scottish Home and Health Department Immunisation against infectious disease 1990. London: HMSO, 1990.

33 SAS System for SunOS, version 8.1. Cary, NC: SAS Institute Inc, 1999.

34 DeWilde S, Carey IM, Bremner SA, et al. Evolution of statin prescribing 19942001: a case of agism but not of sexism? Heart 2003;89:417-21.

35 Statacorp. Stata Statistical Software: release 5.0. 1997. College Station, TX: Stata Corporation.

36 Sharp S, Sterne J. sbe 16: Meta-analysis. Stata Technical Bulletin 1997;38:9-14.

37 Anderson HR, Poloniecki JD, Strachan DP, et al. Immunization and symptoms of atopic disease in children: results from the International Study of Asthma and Allergies in Childhood. Am J Public Health 2001;91:1126-9.

38 Strachan DP. Family size, infection and atopy: the first decade of the "hygiene hypothesis". Thorax 2000;55(suppl 1):S2-10.

39 Completed primary courses at two years of age England and Wales, 19661977, England only 1978 onwards. http://www.hpa.org.uk/infections/ topics_az/vaccination/cover.htm (accessed 26 May 2004).

40 Jansen VAA, Stollenwerk N, Jensen HJ, et al. Measles outbreaks in a population of declining vaccine uptake. Science 2003;301:804.

41 Strachan DP. Hay fever, hygiene, and household size. BMJ 1989;299:1259-60.

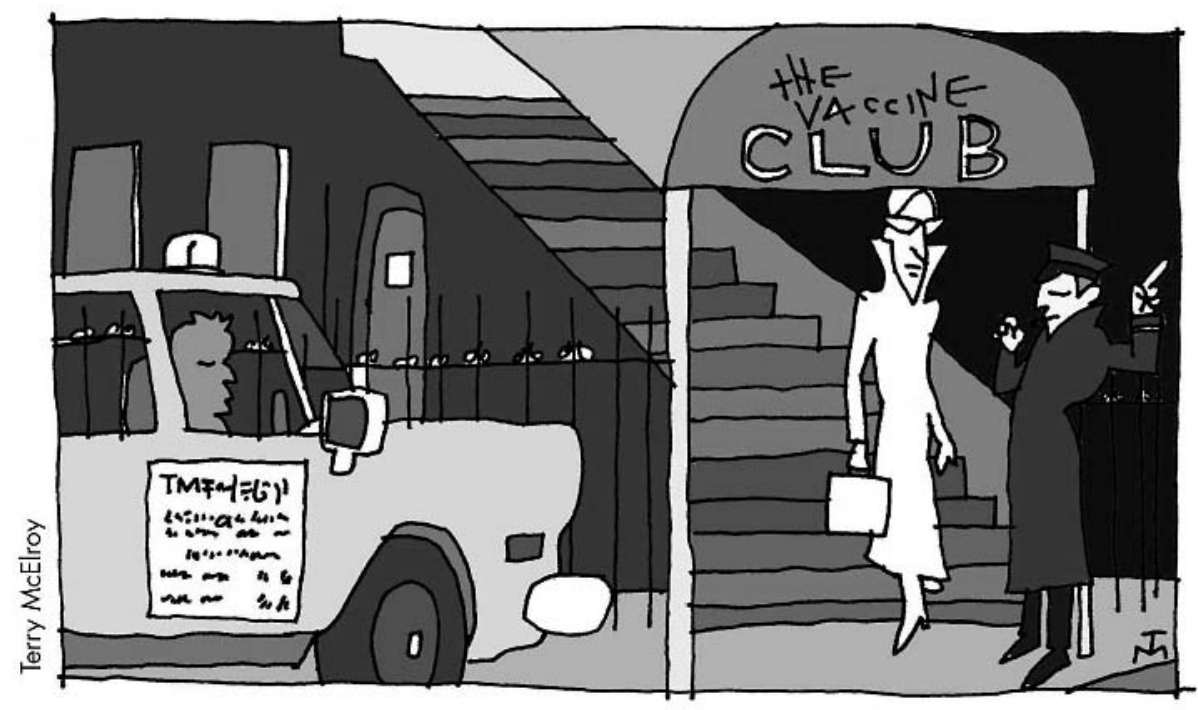

